\title{
Posterior Beta and Anterior Gamma Oscillations Predict Cognitive Insight
}

\author{
Bhavin R. Sheth ${ }^{1,2 *}$, Simone Sandkühler ${ }^{3 *}$, \\ and Joydeep Bhattacharya $a^{2,3,4 *}$
}

\begin{abstract}
Pioneering neuroimaging studies on insight have revealed neural correlates of the emotional "Aha!" component of the insight process, but neural substrates of the cognitive component, such as problem restructuring (a key to transformative reasoning), remain a mystery. Here, multivariate electroencephalogram signals were recorded from human participants while they solved verbal puzzles that could create a small-scale experience of cognitive insight. Individuals responded as soon as they reached a solution and provided a rating of subjective insight. For unsolved puzzles, hints were provided after 60 to $90 \mathrm{sec}$. Spatio-temporal signatures of brain oscillations were analyzed using Morlet wavelet transform followed by exploratory parallel-factor analysis. A consistent reduction in beta
\end{abstract}

\section{INTRODUCTION}

Most daily problems are solved by using sequential reasoning or a "plug-and-chug" approach by applying previously learnt concepts or methods. However, an important subclass of problems can only be solved by an insight (also known as Eureka or "Aha!"), a process by which a problem solver abruptly, through a quantum leap of understanding with no conscious forewarning, moves from a state of not knowing how to solve a problem to a state of knowing how to solve it (Schooler, Fallshore, \& Fiore, 1995; Mayer, 1992). Thus, insight is not an incremental but a transformative step during problem solving, which requires successful restructuring or reformulation of the problem (Duncker, 1945). Cognitive insight is arguably at the core of human intelligence (Hebb, 1949).

How does one characterize insight? One way is to rely on the problem solver's subjective or personal feeling of "Aha!" after they solve the problem; another is to objectively classify a solution on the basis of cognitive processes. For a solution to be classified as insightful, the

\footnotetext{
${ }^{1}$ University of Houston, ${ }^{2}$ California Institute of Technology,

${ }^{3}$ Austrian Academy of Sciences, Vienna, Austria, ${ }^{4}$ University of London, London, UK

*These authors contributed equally to the work.
}

power (15-25 Hz) was found over the parieto-occipital and centro-temporal electrode regions on all four conditions-(a) correct (vs. incorrect) solutions, (b) solutions without (vs. with) external hint, (c) successful (vs. unsuccessful) utilization of the external hint, and d) self-reported high (vs. low) insight. Gamma band $(30-70 \mathrm{~Hz})$ power was increased in right fronto-central and frontal electrode regions for conditions (a) and (c). The effects occurred several (up to 8) seconds before the behavioral response. Our findings indicate that insight is represented by distinct spectral, spatial, and temporal patterns of neural activity related to presolution cognitive processes that are intrinsic to the problem itself but not exclusively to one's subjective assessment of insight.

following three features are key: mental impasse (Fleck \& Weisberg, 2004), restructuring or the creation of a novel and nonobvious representation (Ohlsson, 1992), and parsimony of solution in terms of an economical manipulation of available operators that leads to a dramatic decrease in solution path length (Schilling, 2005).

To understand the neural processes underlying insight, Luo and colleagues presented one-liner riddles to participants in their study and investigated the answer presentation and comprehension period in event-related functional magnetic resonance imaging or fMRI (Luo \& Niki, 2003) and event-related electroencephalogram or EEG (Mai, Luo, Wu, \& Luo, 2004) paradigms. The fMRI study found enhanced activation of the right hippocampus and the EEG study reported more negative postresponse event-related potentials for the "Aha!" condition than for the no-"Aha!" condition in midfrontal cortex. Another study on insight (Jung-Beeman et al., 2004) utilized a remote association task (Bowden \& Jung-Beeman, 2003), which is akin to lexical search. Solutions were classified by the solver's assessment of insight, which was earlier explained to them as a kind of "Aha!" feeling characterized by suddenness and obviousness. EEG revealed a burst of $\sim 40 \mathrm{~Hz}$ gamma frequency band activity in the right temporal brain region beginning $300 \mathrm{msec}$ prior to response.

These pioneering studies revealed interesting neural correlates of the "Aha!" phenomenon; however, each 
had limitations. For instance, firstly, Mai et al. (2004) and Luo and Niki (2003) addressed the neural basis of what we term "outsight," not true insight: Insight is when the solver achieves a solution in his or her own mind (Seifert, Meyer, Davidson, Patalano, \& Yaniv, 1995), whereas outsight is when the person eventually understands the provided solution (as in understanding the punchline of a joke). Therefore, it is possible that insight and outsight have different neural substrates. Secondly, the gamma bursts observed on insightful solutions (Figure 4 in Jung-Beeman et al., 2004) were actually stronger in the postsolution period than in the time period just prior to the solution. Therefore, these gamma bursts possibly reflect affective processes, namely, the underlying joy or sense of relief that typically follows solutions involving "Aha!" (Gick \& Lockhart, 1995), rather than cognitive processes that lead to the solution and the ensuing "Aha!". Thirdly, transformative thinking necessary for insight emerges when two or more conceptually disparate knowledge domains are linked (Terzis, 2001), whereas remote association task calls for verbal fluency and is probably confined to the lexical domain.

Thus, there remains the need for an analysis of neural activity that (a) directly reflects the cognitive processes leading up to a solution (presolution cognitive processes as opposed to postsolution affective processes) of (b) problems that an individual solves internally (true insight as opposed to outsight) by a (c) transformative step of reasoning spanning otherwise unconnected knowledge domains (interdomain as opposed to intradomain associations), including the processes that comprise problem restructuring.

Therefore, in this study, we designed a multivariate EEG study of humans as they attempted to solve openended candidate insight problems that can be solved with a transformative step of reasoning. EEG, with its excellent millisecond resolution, is well suited to analyze the neural activity underlying complex cognitive processes such as problem solving. We analyzed the oscillatory contents of the induced neural activity that directly reflects the cognitive processes leading up to an internally generated solution during problem solving. Large-scale neural oscillations have been implicated in complex cognitive tasks (Grabner, Fink, \& Neubauer, 2007; Krause, Pesonen, \& Hamalainen, 2007; Razumnikova, 2007; Raghavachari et al., 2001; Jausovec \& Jausovec, 2000).

We hypothesize the following. First, we expect that the most robust signature of cognitive insight will be found in the high frequencies as oscillations in the beta $(13-30 \mathrm{~Hz})$ and gamma $(>30 \mathrm{~Hz})$ bands provide a general platform for integration of neuronal information belonging to sensory and cognitive processes (Engel, Fries, \& Singer, 2001; Tallon-Baudry \& Bertrand, 1999). Second, weaker beta band responses in the posterior brain regions corresponding to insight: Transformative thought is characterized by efficient retrieval of informa- tion from various knowledge domains stored in memory and focused concentration or attention to the problem at hand; event-related desynchronization (ERD) of the beta frequencies prominent over parietal areas is observed under conditions of high memory load during encoding and retrieval (Pesonen, Hamalainen, \& Krause, 2007; Pesonen, Bjornberg, Hamalainen, \& Krause, 2006; Bastiaansen, van der Linden, Ter Keurs, Dijkstra, \& Hagoort, 2005). Third, stronger gamma band (>30 Hz) responses in the right frontal brain regions corresponding to insight: Right frontal dominance is observed in the case of open-ended problems that require restructuring (Goel \& Vartanian, 2005)—which is, as described above, a key feature of insight. Fourth, the change in neural activity underlying the transformative thought process must precede the moment of decision or response by several seconds: Insight is likely to consist of both conscious and unconscious cognitive processes (Kihlstrom, 1995); breakdown of the mental impasse and the restructuring that follows the impasse are finally followed by an "Aha!" moment when "everything comes together."

\section{METHODS}

\section{Participants}

Eighteen paid participants (10 men, 8 women; age range $=18-29$ years, mean $=21.2$ years) with no history of sleep or any other neurological disorders were recruited from the student population at the California Institute of Technology.

\section{Stimuli}

Sixteen brainteasers, or verbal puzzles with practical content, were chosen from known problem books and magazines (Gardner, 1978, 1982) (also see supplementary information) as candidate insight problems. Each puzzle was carefully selected with the following criteria:

(1) Specialized knowledge or expertise was not required to solve the puzzles.

(2) There was no set procedure to successfully solve the puzzles-A transformative step of thinking was required. A "plug-and-chug" approach led to a dead end.

(3) Each puzzle was simple to understand; a single insight, and not a series of insights, was required to solve it.

(4) The puzzles were not well known. The participants in our study knew the problems less than $2 \%$ of the time. We discarded these rare trials from our analysis.

(5) Writing tools (pen and paper) were neither required nor provided to solve the puzzles. 
An example puzzle is given here:

Problem: There are three on-off light switches on the wall of the first floor of a building. One of the switches controls an incandescent bulb in a lamp on the third floor of the building. The bulb is initially off. The other two switches do not control the bulb or anything else (they are disconnected). You are allowed to toggle the switches as many times as you want and for as long as you want. How can you find out which one of the three switches turns the light bulb on and off?

The only constraint is that you can walk only once to the third floor to check on the light bulb.

Hint: Keep one of the switches on for an hour and then turn it off.

Solution: You turn the first switch on and leave it on for an hour. Then you turn it off and turn the second switch on, leave the third switch in the off position, and you go upstairs. If the bulb is on, then it's switch number two, which is the one that's on. If the bulb is off and it's cold, then it is switch number three (the switch you never touched) that controls that light. If the bulb is off but it's hot, then it is switch number one.

The default approach toward solving the puzzle is to visually inspect the light bulb, which is likely to fail. Instead, a "multisensory" approach is required to solve the puzzle: Information from the tactile sense must be combined with that from vision. This is, we presume, a critical transformative step of thinking, making puzzles such as this ideal for the study of insight (Sternberg \& Davidson, 1995; Metcalfe \& Wiebe, 1987).

\section{Behavioral Procedure}

Problems were presented on a Macintosh G4 running MATLAB and EEG recordings were stored on a PC. The two computers were connected via a serial port, allowing event triggers to be marked on the EEG record. The participant sat comfortably in a chair at a fixed distance from the monitor on which the problem was presented. Upon solving the problem, the participant had to provide an insight rating (10: maximum feeling of insight; 0: no feeling of insight). Prior to the experiment, the participant was told that a feeling of insight is a kind of "Aha!" characterized by suddenness and obviousness of the solution (Jung-Beeman et al., 2004). Prior to introduction of the test puzzles, three practice puzzles helped familiarize participants with the procedure.

Figure 1 shows the flowchart of a single trial. In brief, a trial began with the participant hitting a keyboard key designated as "go." This caused the puzzle statement to appear and the participant had $30 \mathrm{sec}$ to read it.
Afterward, a question statement was presented and the participant had 60 to $90 \mathrm{sec}$ to solve the puzzle. If the participant could not produce a solution within this prescribed time, a hint appeared and another 60 to $90 \mathrm{sec}$ was allowed to solve the puzzle. The hint was an implicit or first-order hint (Gick \& Lockhart, 1995), which provided some general direction but did not reveal the solution. In essence, the hint, if successfully utilized, constrained the search process to a subspace of the problem space.

We asked participants not to mentally check their proposed solution but rather to respond as soon as a solution emerged; this isolated the moment of subjective insight as much as possible. Participants hit a designated "stop" key as soon as they solved the puzzle within the prescribed time. Then, they provided a rating of insight and verbalized their proposed solution. The experimenter verified its correctness. Breaks were provided between puzzles.

\section{EEG Recordings}

EEG signals were collected using a 32-channel EEG system (ActiveTwo, BioSemi Inc.). Electrodes were placed using the extended International 10-20 System (Jasper, 1958). The sampling frequency was $256 \mathrm{~Hz}$. EEG signals were recorded with a band-pass filter setting of 0.16 to $100 \mathrm{~Hz}$. EEG signals were algebraically re-referenced against averaged earlobes and were divided into three segments: (i) initial 30-sec time period from puzzle onset, (ii) intermediate 20 -sec time period containing hint (10 sec before and after hint presentation), and (iii) final 31-sec time period (30 sec before the response followed by $1 \mathrm{sec}$ ). We utilized an extended temporal Independent Component Analysis (ICA) algorithm to remove eye artifacts from these EEG segments in a semiautomated manner (Nicolaou \& Nasuto, 2003). The criteria for rejection of components were: (i) sparsely distributed ICA components, (ii) short-range linear trends, and (iii) nonstationary time courses of the standard deviations of the ICA components. Afterward, we visually screened the signals and manually edited the contaminated ICA components. Any segment still containing amplitudes exceeding $80 \mu \mathrm{V}$ was removed from further analysis. Four individuals were removed due to excessive artifacts or disinterest in the experiment.

Depending on solution, utilization of hint, and the participant's own insight rating, we categorized experimental trials into eight conditions as follows: (i) correct solution (HIT), (ii) incorrect or false-positive solution (FP), (iii) successful hint (SH) leading to a correct solution, (iv) unsuccessful hint (UH) leading to an incorrect solution or miss, (v) subjective high rating (HR) of insight, (vi) subjective low rating (LR) of insight, (vii) hit with no prior hint (nH_HIT), and (viii) posthint hit (H_HIT). 
Figure 1. The flowchart of a single trial. The hint is presented only after the individual fails to solve the puzzle in the prescribed time.

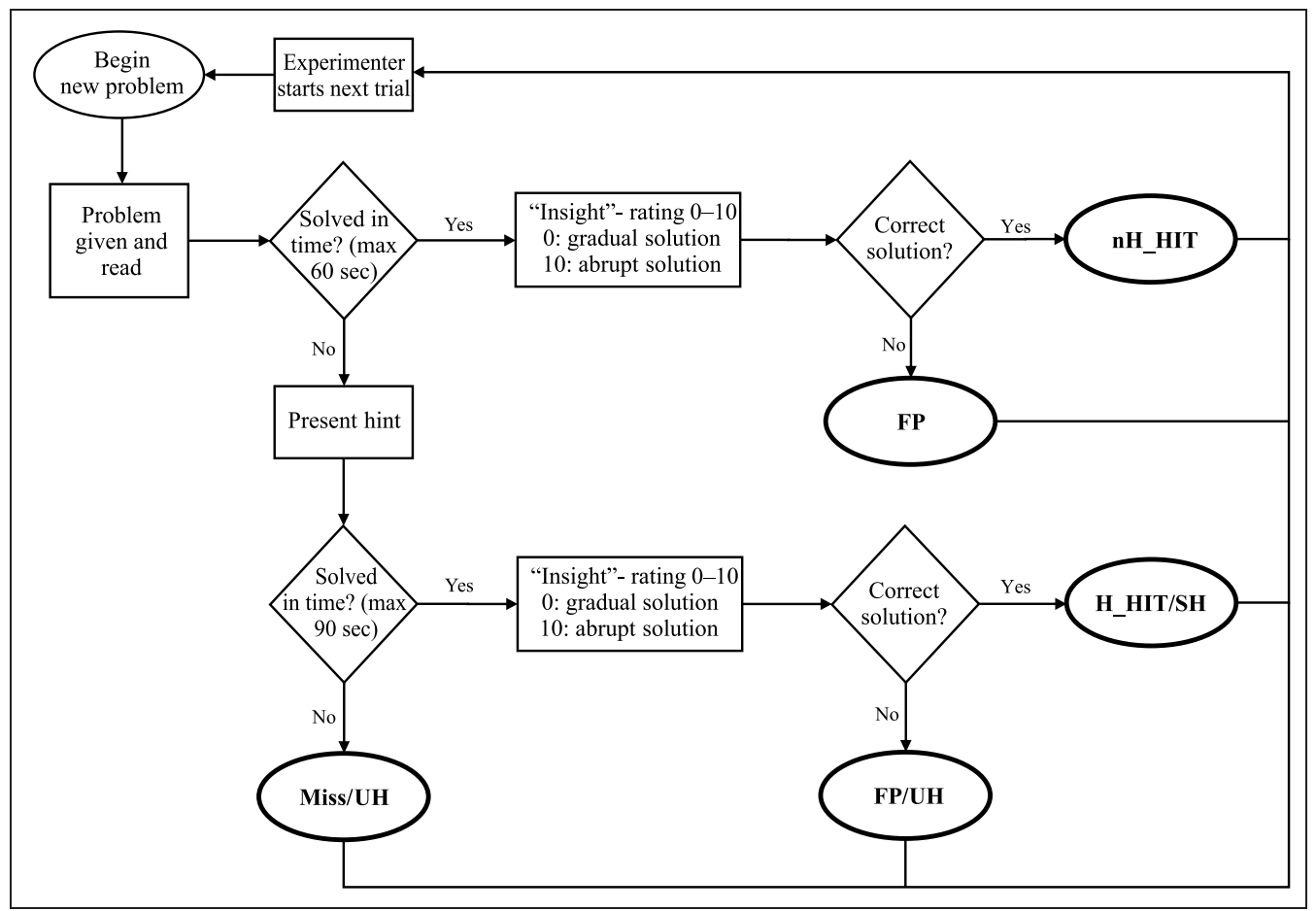

\section{Data Analysis}

To estimate the time-varying spectral content in the EEG signal, the original signal $x(t)$ was convolved with scaled and translated complex Morlet wavelets $\Psi_{\omega}(t)$ with a Gaussian shape in both the time $\left(S D=\sigma_{t}\right)$ and the frequency domains $\left(S D=\sigma_{f}\right)$ around frequency

$$
\omega: \Psi_{\omega}(t)=\exp \left(-\frac{t^{2}}{2 \sigma_{t}^{2}}\right) \exp (i 2 \pi \omega t)
$$

The wavelet family is characterized by a constant ratio $\omega / \sigma_{f}=7$, with $\sigma_{f}=1 /\left(2 \pi \sigma_{t}\right)$. The center frequency $\omega$ ranged from 1 to $70 \mathrm{~Hz}$ in 1-Hz steps. Thus, all standard EEG frequency bands, namely, delta, theta, alpha, beta, and gamma, were analyzed. The time-varying energy of the signal at time $\xi$ and frequency $\omega, E(\xi, \omega)$ is defined as $E(\xi, \omega)=\left|\left(\Psi_{\omega} \otimes x\right)(\xi)\right|^{2}$, where $\otimes$ represents the convolution operator.

The first 2 sec of the statement reading time period (see Figure 1) were used as a baseline. For each participant, the mean ratio $R(\xi, \omega)$ in decibels between the energy of the trials and that of the baselines was given by:

$$
\begin{aligned}
R(\xi, \omega)= & 1_{0} \log _{10}\left[\left(\prod_{i=1}^{N} E_{i}(\xi, \omega)\right)^{1 / N}\right] \\
& -10 \log _{10}\left[\left(\prod_{j=1}^{M} \overline{\mathrm{e}}_{j}(\omega)\right)^{1 / M}\right],
\end{aligned}
$$

where $N$ is the total number of trials, $M$ the total number of baselines and $\overline{\mathrm{e}}_{j}(\omega)$ is the mean energy of the 2-sec baseline period of baseline $j . R$ indicates the amount of increase (or decrease) in the oscillatory content of induced EEG brain responses during task/trial-related processing with respect to baseline. Because we selected artifact-free baseline and task segments separately, $N$ and $M$ could be unequal.

\section{Task Comparisons}

Time-frequency representation (TFR) analyses with baseline corrections were performed for all eight conditions and then we conducted four pairwise comparisons of conditions. One of the two conditions in each comparison engaged more strongly transformative thought processes. Whereas the EEG signals underlying each comparison are unique, we reasoned that the shared features among them would be reliable and generalizable neural signatures of transformative reasoning.

The four comparisons are the following. (1) HITs versus FPS: We contrasted neural TFRs over a 10-sec pre-response period for trials on which the participant generated a correct, insightful solution (HIT) versus trials on which the participant generated an incorrect or false positive solution (FP). It should be noted that the participant did not know if their proposed solution was correct or not at the time of response (button press) but knew only after he or she had verbalized it and the experimenter validated it. For this comparison, HIT trials featured true transformative reasoning. (2) nH_HIT versus H_HIT: Insight problems typically contain 
open-ended, unstructured information. Restructuring a problem in a way different from before is a key first step in the process leading up to the insight. Restructuring is internal or can be guided by external hints that constrain the problem space. We provided a hint when the participant failed to solve the puzzle in the prescribed time, and contrasted EEG spectral content during the 10-sec preresponse period on two subclasses of HIT trials: trials on which participants came up with the correct solution by themselves (nH HIT, successful restructuring without the aid of a hint) and HIT trials on which the participants correctly solved the puzzle after the experimenter provided a hint (H_HIT, successful restructuring with the aid of a hint). For this comparison, trials on which participants restructured problem information on their own (nH_HIT) contained a core component of transformative reasoning. (3) SH versus UH: We contrasted EEG spectral content during the 10-sec prehint and 10-sec posthint periods between trials on which, following the external hint, participants hit upon the correct solution (successful hint trials or $\mathrm{SH}$ ), and trials on which they produced either an incorrect, noninsightful solution or no solution at all (unsuccessful hint trials or UH). For this comparison, $\mathrm{SH}$ trials featured (correct) transformative reasoning. (4) "Aba!" versus "non-Aba!': Upon responding, participants indicated whether they had solved the problem with insight (rating $\geq 8$ ) or without (rating $\leq 3$ ) on a 10point scale (see Methods). It is to be noted that a problem solver might rate an incorrect solution as highly insightful or a correct solution as noninsightful: 38\% (21/55) of FPs were "Aha!" solutions and 33\% (17/50) of HITs were "non-Aha!" solutions. We selected features of the TFR corresponding to transformative thought that were consistently observed on the above comparisons.

For each of the four pairs of comparisons, statistical tests (two-sided) were performed between the timefrequency contents on each electrode region of the selected two conditions. Statistical analysis was performed with windows spanning $500 \mathrm{msec} \times 2 \mathrm{~Hz}$ in the timefrequency domain with a $250 \mathrm{msec} \times 1 \mathrm{~Hz}$ overlap. Because the number of epochs varied across participant and condition, we assumed that the original observations $E_{i}(\xi, \omega)$ and $\overline{\mathrm{e}}_{j}(\omega)$ were homoscedastic, as the variances of the spectral estimators got smaller with increasing number of epochs. To incorporate this feature into the statistical analysis, we used a more robust, heteroscedastically consistent paired quasi- $t$ test (Zeileis, 2004).

\section{PARAFAC Analysis}

For each comparison, the statistical analysis resulted in a three-dimensional statistical ( $F$ values) time-frequency maps of Electrode $\times$ Frequency $\times$ Time. The statistical maps were subsequently decomposed by parallel-factor analysis (PARAFAC) (Morup, Hansen, Herrmann, Parnas, \& Arnfred, 2006), which selects the components of the map that explain the largest amount of signal variance. That is to say, PARAFAC zooms in on the scalp topography, spectral frequency, and temporal dynamics that provide maximum discriminatory power between two compared conditions. Using PARAFAC analysis, we summarized the time-frequency energy variations, which were expressed in $t^{2}$ test values, by decomposing them into Space (or electrode) $\times$ Frequency $\times$ Time atoms The $t^{2}$ test values for each channel were combined in a three-way array $X(I \times J \times K)$ of Space $\times$ Frequency $\times$ Time and the PARAFAC model was formulated as:

$$
x_{i j k}=\sum_{f=1}^{F} a_{i f} b_{j f} c_{k f}+\varepsilon_{i j k}
$$

where $F$ is the number of factors. This formula corresponds to the assumption that a given frequency signature $b_{f}$ of strength $c_{f}$ in time has been assorted in the electrodes by $a_{f}$. Due to the fact that PARAFAC vector loadings are unique to within a factor of scale and permutation, we normalized each vector to a maximum value of 1 . The PARAFAC model is related to higherdimensional principal component analysis, but offers better statistical properties (uniqueness, rotation etc.) than principal component analysis.

Parameters of the PARAFAC model fit were estimated by minimizing the squared error of the fitted model with an alternating least squares algorithm (Bro, 1998). We ensured that a stable solution was reached by using random orthogonalized values for initialization and by repeating the algorithm three times followed by visual comparison of the results. The optimum number of factors was determined by core consistency diagnostic, the sum-squared error, number of iterations, and 13-fold cross-validation (Bro, 1998; see supplementary Figure 1). It might be noted that the PARAFAC model was applied on $F$ values, not on the raw TFR values. This approach emphasizes the most robust differences between two compared conditions, and thus, more localized spatial maps are likely. Other effects, which are common to both conditions (see Figure 5 of Morup et al., 2006 for an illustration of this approach) cancel out as a result.

\section{RESULTS}

For each pair of comparisons (see Methods), our analysis consisted of three steps: (i) estimating time-frequency structure of the induced oscillatory activity for both conditions, followed by (ii) investigating their statistical differences in terms of $F$ test values, and finally, (iii) applying PARAFAC analysis on these $F$ values.

We briefly describe here these steps for the comparison HIT versus FP. Figure 2 shows the difference (HIT minus FP) TFR plots for all 30 electrode locations. As compared to FP responses, HIT responses show two 


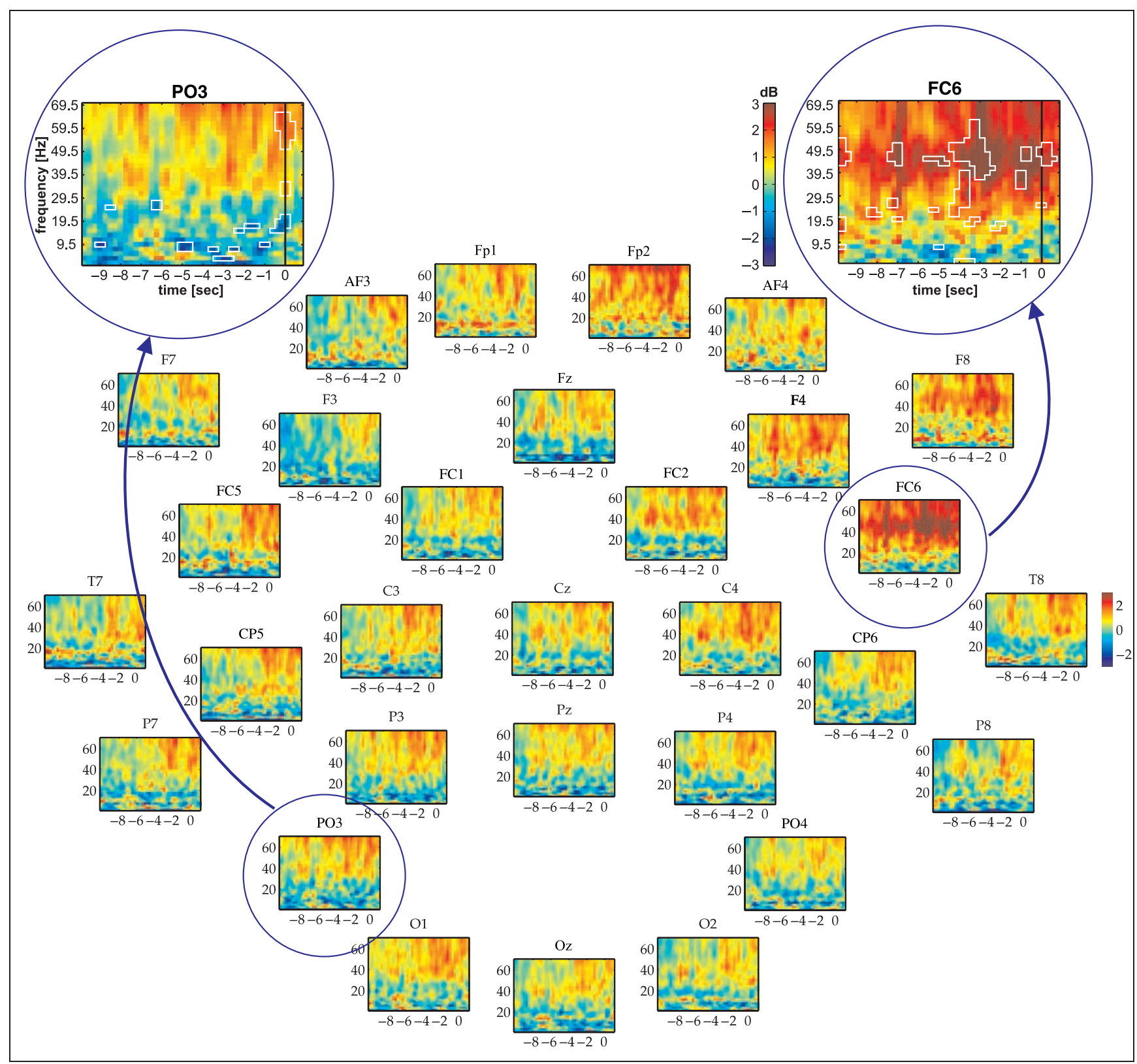

Figure 2. Morlet wavelet based TFRs of the induced oscillatory activity for correct (HIT) minus incorrect (FP) responses. The $x$-axis represents time, the $y$-axis represents frequency. All 30 electrodes are shown with their locations mentioned on top of each subplot. Red (blue) indicates increased spectral power for HIT (FP) and white rectangles denote zones of significance $(p<.05)$. Moment of response is at $t=0$. The two magnified TFR plots depict the most conspicuous gamma band effect and beta band effect at electrode locations FC6, right fronto-central, and $\mathrm{PO} 3$, left parieto-occipital, respectively.

robust effects: decrease in beta band activity and increase in gamma band activity, and these effects are most prominent at FC6 and PO3, respectively (note also the white rectangles indicating the zones of statistical significance as determined by the $F$ values). Next, these three-dimensional data (Electrode $\times$ Time $\times$ Frequency) of $F$ values are decomposed by PARAFAC analysis into the sum of four factors (see supplementary figure), where each factor has three modes: spatial, temporal, and spectral (see Methods). We find that the earlier electrode locations (FC6, PO3) also appear to prominence in the factors (Factor 1 and Factor 4). Other similarities can be observed as well, for example, strong alpha suppression, for HIT responses, in the TFR plot at electrode Fz at around $t=-5 \mathrm{sec}$ and a very localized profile with similar space-time-frequency signatures for Factor 3.

We show another example on linking a factor with actual TFR representations. For the comparison nH_HIT versus H_HIT, Factor 3 shows a spatial mode with sharp localization around F8 (see supplementary figures), and its temporal and spectral modes are displayed in Figure $3 \mathrm{~B}$ and $\mathrm{C}$, respectively. The zone of significance in the TFR plots of F8 is shown in Figure 2A and is remarkably consistent with the two modes as described earlier. 


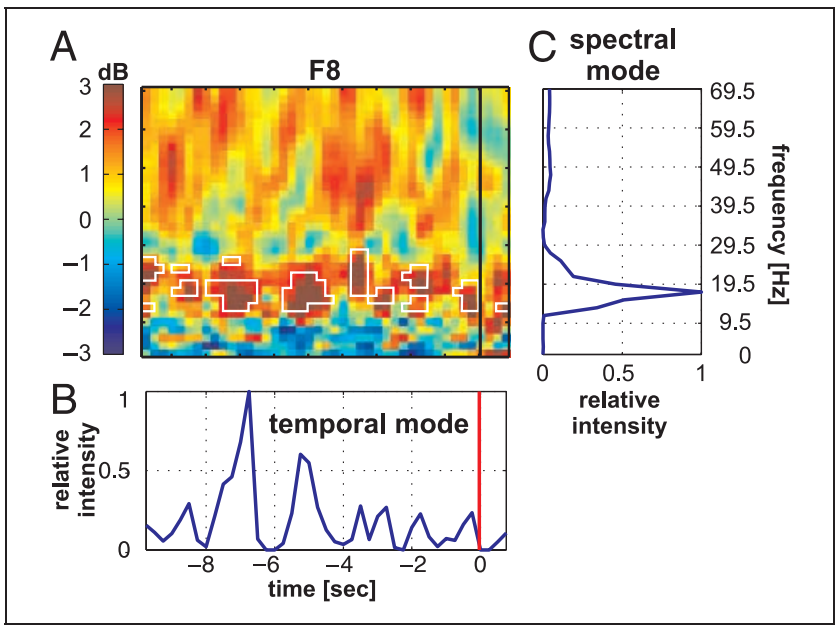

Figure 3. Linking PARAFAC analysis with individual TFRs. (A) TFR of the induced oscillatory activity at electrode location F8 for correct responses without hint (nH_HIT) minus correct responses with hint (H_HIT). The white rectangles denote the zones of significance $(p<.05)$. (B) The temporal mode and (C) the spectral mode of one of the factors produced by the PARAFAC decomposition for the identical pair of comparison, nH_HIT versus H_HIT (see supplementary figure for details). Note the time and spectral information in A is essentially captured by B and C, respectively.
These all together demonstrate the efficacies of PARAFAC analysis on $F$ values to identify the region of interest in a higher-dimensional dataset.

Next, we applied the PARAFAC analysis on all four comparisons, and not surprisingly, it yielded features unique to each comparison (supplementary figures). However, we strategically focused only on those features that were common and consistent, in terms of frequency band and the associated ERS/ERD changes, across all comparisons. Because transformative reasoning is also common across all comparisons, this cognitive process is likely to be reflected by the earlier common neural features.

As hypothesized in the Introduction, there was a decrease in spectral power in the beta band in the parietal and parieto-occipital areas on all four comparisons. Factor 4 of the signal variance on the HIT versus FP trials was dominated by differences in the left parietooccipital region (PO3; Figure $4 \mathrm{~A}$ ) in three distinct frequency bands, but significantly reduced power for HIT trials was found only in the beta frequency band [15$20 \mathrm{~Hz}$; FP $>$ HIT, $F(1,11)=10.39, p<.0042]$. Factor 2 of the signal variance on the $\mathrm{nH}$ HIT versus H HIT trials showed steady, significantly reduced power on the nH_HIT trials as compared to the H_HIT trials in the
Figure 4. Decrease in spectral power in the beta frequency band in the parietal and parieto-occipital regions corresponding to transformative thought. The spatial (leftmost panel), spectral (middle panel), and temporal (rightmost panel) modes of the PARAFAC decomposition of $F$ values highlighting decrease in beta power are depicted between conditions of the following four comparisons (A) HIT vs. FP, (B) nHIT vs. H_HIT,

(C) SH vs. UH, and (D) "Aha!" vs. "non-Aha!" (see supplementary figures for a complete description of the main components of the PARAFAC model). The percentages on the left denote the proportion of the variance accounted for by the particular component for the given pairwise comparison. The red line in the temporal mode panel represents the instant participants pressed the button to propose a solution; the green line represents the instant at which the hint was presented. The intensities represent the two-sided statistical significance of the mode.
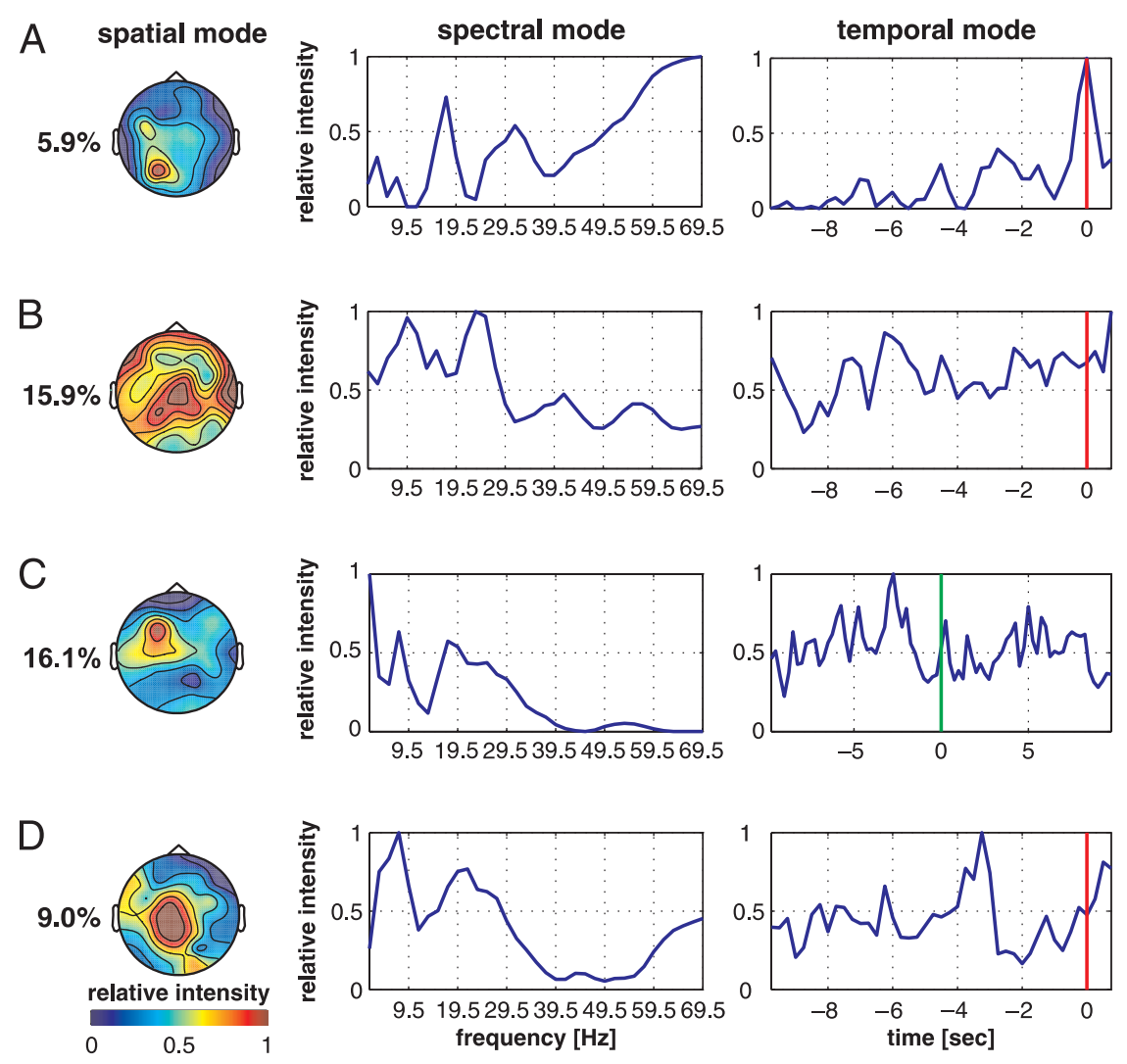
Figure 5. Change in spectral power in the gamma frequency band corresponding to transformative thought. The spatial (leftmost panel), spectral (middle panel), and temporal (rightmost panel) modes of the PARAFAC decomposition of $F$ values highlighting changes in gamma power are illustrated between conditions of the following comparisons (A) HIT vs. FP, (B) nHIT vs. H HIT, (C) $\mathrm{SH}$ vs. UH, and (D) "Aha!" vs. "non-Aha!".
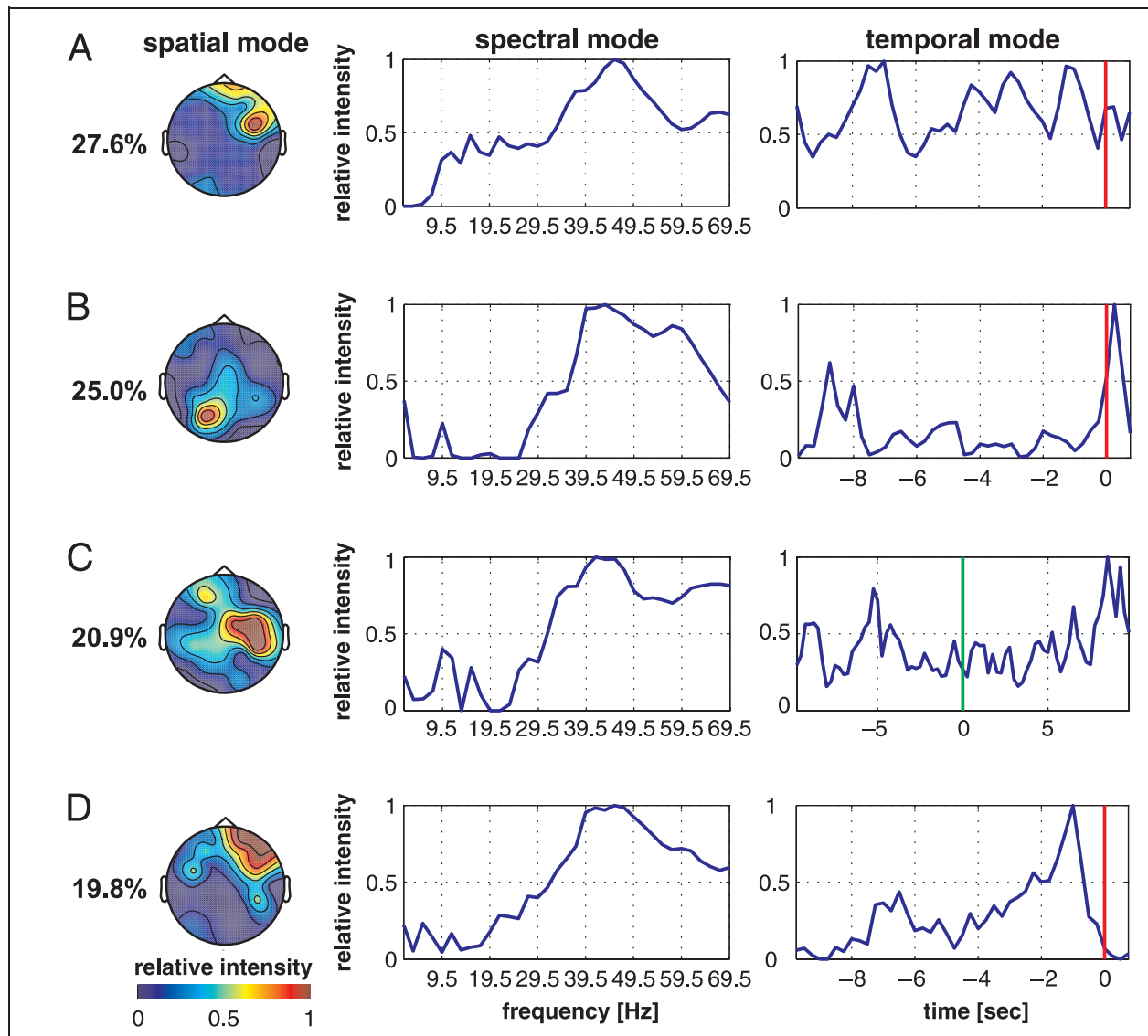

upper beta $\left[f_{c}=23.5 \mathrm{~Hz}, p<.004\right.$ (at $\mathrm{Cz}$ and $t=$ $-5.75 \mathrm{sec})]$ and lower-frequency bands $\left(f_{c}=9.5 \mathrm{~Hz}\right.$, $p<.014, t=-6.25 \mathrm{sec}$ ) over a wide expanse of the brain, but mainly over the right parieto-central and temporal regions (Figure 4B). Factor 2 of the signal variance on the $\mathrm{SH}$ versus $\mathrm{UH}$ trials showed several differences, among which was significantly reduced power in the left frontal regions (mainly F3) in the beta $\left(f_{c}=17.5 \mathrm{~Hz}\right.$, $p<.002, t=-2.5 \mathrm{sec}$ ) frequencies (Figure 4C). Factor 3 of the signal variance on the "Aha!" versus "non-Aha!" trials was a significant reduction in power in the beta frequency band in the central-parietal regions ("nonAha!" > "Aha!", Pz, $f_{c}=21.5 \mathrm{~Hz}, p<.0001$; Figure 4D); there was also significant increased power in the lower alpha $\left(\mathrm{C} 3, f_{c}=7.5 \mathrm{~Hz}, p<.007\right)$ frequency band for "Aha!" as compared to "non-Aha!" solutions.

We also observed a change in gamma power in the right anterior region as hypothesized, although the results were somewhat less consistent than the beta band decrease. Factor 1 of the difference in HITs versus FPs showed a conspicuous increase for HIT trials in the right fronto-central region [FC6; HIT $>$ FP, $F(1,11)=$ $6.75, p<.025]$ in the gamma frequency $\left(f_{c}=45.5 \mathrm{~Hz}\right)$ range (Figure 5A). Similarly, Factor 1 of the difference between "Aha!" and "non-Aha!" was significant over the right frontal region ["Aha!" > "non-Aha!," $f_{c}=39.5 \mathrm{~Hz}$, F4 $(p<.008)$; F8 $(p<.002, t=-1.25$ sec $)$; Figure 5D] in the broad gamma frequency band $(30-70 \mathrm{~Hz})$. Factor 1 of the difference in neural activity between $\mathrm{SH}$ and $\mathrm{UH}$ depicted a gradually increasing difference in the gamma band $(31-70 \mathrm{~Hz})$ starting from hint-onset over right centro-temporal regions (Figure $5 \mathrm{C}$ ), but the direction of the effect was opposite [UH $>$ SH; CP6: $F(1,11)=$ 11.04, $p<.0035$; FC2: $p<.03$; C4: $p<.023$ ] compared with the previous comparisons. Factor 1 of the comparison nH_HIT versus H_HIT indicated greater power exclusively in the gamma band $\left(f_{c}=43.5 \mathrm{~Hz}\right)$ for the condition that contains more transformative thought (nH_HIT), and the difference was over the left parietoOccipital region $\left(\mathrm{PO} 3\right.$; $n \mathrm{nH}_{-} \mathrm{HIT}>\mathrm{H}_{-} \mathrm{HIT}, p<.006$; Figure $5 \mathrm{~B}$ ). In summary, there was an increase in broad band gamma power in the right fronto-central and frontal electrode regions on two of the four comparisons but the results were not as consistent as the beta band decrease in terms of brain region and direction of change (increase/decrease) in spectral power (see Discussion).

\section{DISCUSSION}

Our EEG study of the neural basis of insight and transformative thinking during problem solving has yielded results that hold across a diverse range of conditions and comparisons. 
As hypothesized, there was a decrease in beta band (13-30 Hz) power in the parietal, parieto-occipital, and centro-temporal brain regions when the person was predominantly engaged in transformative reasoning. Cognitive processing is sometimes associated with change in beta power (Pesonen et al., 2007; Bastiaansen et al., 2005; Weiss \& Rappelsberger, 1998). Further, decreased beta power in the parietal and parieto-occipital areas is seen in conjunction with modality-independent increase in memory load (Pesonen et al., 2006, 2007), which is believed to be necessary for transformative thought. Our results thus strongly suggest a prominent role for beta oscillations in transformative reasoning tasks that critically involve semantic memory.

Enhanced power in the gamma band $(30-70 \mathrm{~Hz})$ and increased involvement of the right cerebral hemisphere (right frontal, fronto-central, and centro-temporal regions) were also observed when participants correctly solved the insight problem and when they reported experiencing "Aha!" The right prefrontal cortex is activated on problems requiring set-shift transformation (Goel \& Vartanian, 2005; Miller \& Tippett, 1996), which is a type of restructuring, and on tasks involving sequential thinking when a belief-logic conflict causes a change in the reasoning process (Goel, Buchel, Frith, \& Dolan, 2000). Further, the right hemisphere appears to help make available a set of alternative and less probable word meanings in a lexical task (Coney \& Evans, 2000), which corresponds to the generation of multiple solutions in insight problems. Curiously, gamma power was lower on successful (hit) trials than unsuccessful trials in the immediate time period following hint presentation. One account is that the hint constrained the search space to the point that the posthint period did not require true transformative reasoning on some trials. The posthint comparison was the only one of four comparisons that did not include the moment of response and an alternative account is that enhanced gamma power may be a neural correlate of the moment of subjective insight but not of the restructuring and other processes of transformative reasoning that precede it. Moreover, the differences in spatial location of the increase (FC6, F8) versus decrease (CP6, C4) in gamma power suggest the presence of two distinct gamma response systems. Additional experiments are needed to resolve these issues. Nonetheless, on the basis of past and present findings, we can reasonably claim that the right prefrontal region and gamma band oscillations are engaged in transformative reasoning.

Of critical importance, the present results support our fourth and final hypothesis, namely, that the change in oscillatory activity underlying transformative reasoning represents unconscious cognitive, not affective, processes: In contrast to the fact that affective "Aha!" processes typically occur around the moment of response (JungBeeman et al., 2004), changes in spectral power corresponding to transformative thinking in our study occurred several seconds prior to response. Examples include enhanced gamma band activity for HIT versus FP solutions in the right fronto-central brain region $8 \rightarrow 1 \mathrm{sec}$ prior to the response (Figure $3 \mathrm{~A}$ ), decreased beta band activity on nH_HIT versus H_HIT trials $6 \mathrm{sec}$ prior to the response (Figure 2C), and enhanced upper alpha $(13.5 \mathrm{~Hz})$ activity in the right parieto-occipital region $7 \mathrm{sec}$ before presentation of the hint on $\mathrm{SH}$ versus $\mathrm{UH}$ trials (Factor 3 in Supplementary Figure 3). Insight problem solvers are usually unable to report the internal mental processes that precede their insightful solution (Ohlsson, 1992) or be joyful before solving the problem on hand; therefore, the changes in oscillatory rhythms that occur well before the conscious response are unlikely to correspond to feelings of joy or relief that accompany problem solution but rather to subconscious (and possibly automatic) mental recombinations of apparently distant yet remotely connected representations prior to solution (Simonton, 1999; Seifert et al., 1995; Metcalfe \& Wiebe, 1987)

Our findings also lend support to the proposal that prior brain state, namely, the level of mental preparedness, contributes to later restructuring of the problem representation: There was a difference in brain activity even before the hint was presented between puzzles that were eventually solved and those that were not. Unlike in Kounios et al. (2008), no significant differences were observed over the time period before problem presentation; differences were observed over the time period after problem presentation but before presentation of the hint. This effectively argues for the existence of a brain state that is receptive to the incorporation of new information (hint) with an existing cognitive representation.

Thus far, we have focused on commonalities in oscillatory patterns of brain activity across the four comparisons. Clearly, there are also patterns that are unique to each (Supplementary Figures 2-5). For instance, gamma band activity in the right parieto-occipital region is greater for "Aha!" versus "non-Aha!" solutions (Supplementary Figure 5). This result is compatible with reports of emotionally loaded stimuli enhancing gamma band activity in the right parietal region (Aftanas, Reva, Varlamov, Pavlov, \& Makhnev, 2003; Keil et al., 2001), similar findings dissociating affective from cognitive processes in the context of a study of neural correlates of humor (Goel \& Dolan, 2001), and with our point that the reasoning leading up to the insight and the resulting "Aha!" do not have identical neural substrates.

Let us offer a few critical remarks. First, our participants moved their hand to press a button upon solving a problem. Beta ERD is often reported as a neural correlate for movement preparation and execution; therefore, one might argue that our principal finding of beta ERD corresponds to preparatory motor activity rather than transformative reasoning. However, both conditions of each pairwise comparison either concluded with an identical button press (HITs vs. FPs, nH_HITs 
vs. H_HITs, HRs vs. LRs) or were marked by a common event (i.e., hint in SH vs.UH). Thus, preparatory motor activity was either common within a given comparison, or absent altogether. Also, preparatory motor activity, or the readiness potential as it is called sometimes, is mainly represented by frequencies below $3 \mathrm{~Hz}$ (Libet, Gleason, Wright, \& Pearl, 1983; Kornhuber \& Deecke, 1965), whereas our effects were mainly in the higher beta $(21-30 \mathrm{~Hz})$ and gamma frequencies $(>30 \mathrm{~Hz})$. In sum, beta ERD cannot correspond to motor preparation. Second, our choice of analysis measure needs clarification. One way of analyzing high-dimensional data such as ours is to treat each channel, frequency, and time instant in isolation and statistically measure the reliability of the pairwise difference in signal at each point of this high-dimensional space separately. Controlling for multiple comparisons, one will typically obtain isolated points in time, space, and frequency that significantly differ between the two conditions being tested. Given that there is no a priori hypothesis that is so specific in time, frequency, or space, measurement resolution per se is not so precise, and time instants, frequencies, and especially electrodes are not isolated, independent units, it is more reasonable to think about the neural correlate of transformative reasoning in terms of clusters within high-dimensional space instead. PARAFAC is essentially a data exploratory tool (Morup, Hansen, \& Arnfred, 2007) whose strength lies in identifying regions where one might find differences in space, time, and frequency between two conditions. Raw $F$ statistics were input to the PARAFAC analysis to identify clusters of statistically significant regions in this space. The results of the PARAFAC analysis need to be validated with the raw time-frequency plots, which we did (Figures 1, 2, and 3). In order to cross-validate our PARAFAC findings, we reanalyzed our raw TFR data on isolated units of frequency and space with a multivariate statistical test (Hemmelmann, Horn, Süsse, Vollandt, \& Weiss, 2005) based on false discovery rate (Benjamini \& Hochberg, 1995), which corrects for multiple comparisons; again, beta band effects emerged as the most prominent statistically significant characteristic of transformative reasoning among the four comparisons. In sum, PARAFAC is a useful, powerful way of isolating significant portions of high-dimensional EEG data. Third, EEG has poor spatial resolution; results localizing function to discrete brain areas must be interpreted with caution. Moreover, the involvement of subcortical brain structures, such as the hippocampus, in insight problem solving (Luo \& Niki, 2003) is underemphasized in the EEG. Fourth, each pairwise comparison is unique in some ways. Therefore, one must be cautious before claiming beta and gamma oscillations are the neural substrate of insight. On the other hand, one can reasonably claim that the effects are more consistent and robust in these frequency bands. Finally, our puzzles vary in cognitive content; nonetheless, all share one element: Each requires transformative thought.
In conclusion, our findings indicate that insight is a distinct spectral, spatial, and temporal pattern of unconscious neural activity corresponding to presolution cognitive processes, and not to one's self-assessment of their insight or the emotional "Aha!" that accompanies problem solution. Future studies will determine the generality of our findings to other classes of problems and how modulations of neural activity patterns by clever behavioral or brain computer interface manipulations affect insightfulness.

\section{Acknowledgments}

The research was supported by a GEAR grant from the University of Houston (B. R. S.), Cline Discovery Grant-Caltech (B. R. S.), JST.ERATO Shimojo project (J. B.), and ÖNBJubiläumsfondprojekt (J. B.). J. B. also thankfully acknowledges the support of the Sloan-Swartz Foundation. PARAFAC models and diagnostics were calculated using "The N-way toolbox" for MATLAB kindly provided by Claus Andersson and Rasmus Bro (www.models.kvl.dk/source/nwaytoolbox). We thank Harald Stögbauer for the programs on artifact removal, Larry Varghese for the verbalized protocol analysis, and Vinu Ipe for help in finding appropriate puzzles.

Reprint requests should be sent to Bhavin R. Sheth, Department of Electrical and Computer Engineering, University of Houston, N308, Houston, TX 77204-4005, or via e-mail: brsheth@uh.edu.

\section{REFERENCES}

Aftanas, L. I., Reva, N. V., Varlamov, A. A., Pavlov, S. V., \& Makhnev, V. P. (2003). Analysis of elicited EEG synchronization and desynchronization in emotional activation: Temporal and topographic characteristics. Zhurnal Vyssheï Nervoï Deiatelnosti Imeni I P Pavlova, 53, 485-494.

Bastiaansen, M. C., van der Linden, M., Ter Keurs, M., Dijkstra, T., \& Hagoort, P. (2005). Theta responses are involved in lexical-semantic retrieval during language processing. Lournal of Cognitive Neuroscience, 17, 530-541.

Benjamini, Y., \& Hochberg, Y. (1995). Controlling the false discovery rate: A practical and powerful approach to multiple testing. Journal of the Royal Statistical Society: Series B, 57, 289-300.

Bowden, E. M., \& Jung-Beeman, M. (2003). Aha! Insight experience correlates with solution activation in the right hemisphere. Psychonomic Bulletin E Review, 10, 730-737.

Bro, R. (1998). Multi-way analysis in the food industry: Models, algorithms and applications. Amsterdam: University of Amsterdam (NL) and Royal Veterinary and Agricultural University (DK).

Coney, J., \& Evans, K. D. (2000). Hemispheric asymmetries in the resolution of lexical ambiguity. Neuropsychologia, 38, 272-282.

Duncker, K. (1945). On problem-solving. Psychological Monographs, 58, ix, 113.

Engel, A. K., Fries, P., \& Singer, W. (2001). Dynamic predictions: Oscillations and synchrony in top-down processing. Nature Reviews Neuroscience, 2, 704-716.

Fleck, J. I., \& Weisberg, R. W. (2004). The use of verbal protocols as data: An analysis of insight in the candle problem. Memory \& Cognition, 32, 990-1006.

Gardner, M. (1978). Aha! Aba! insight. New York: Scientific American. 
Gardner, M. (1982). Aha! Gotcha: Paradoxes to puzzle and delight. San Francisco: W.H. Freeman.

Gick, M. L., \& Lockhart, R. S. (1995). Cognitive and affective components of insight. In R. J. Sternberg \& J. E. Davidson (Eds.), The nature of insight (pp. 197-228). Cambridge, MA: MIT Press.

Goel, V., Buchel, C., Frith, C., \& Dolan, R. J. (2000). Dissociation of mechanisms underlying syllogistic reasoning. Neuroimage, 12, 504-514.

Goel, V., \& Dolan, R. J. (2001). The functional anatomy of humor: Segregating cognitive and affective components. Nature Neuroscience, 4, 237-238.

Goel, V., \& Vartanian, O. (2005). Dissociating the roles of right ventral lateral and dorsal lateral prefrontal cortex in generation and maintenance of hypotheses in set-shift problems. Cerebral Cortex, 15, 1170-1177.

Grabner, R. H., Fink, A., \& Neubauer, A. C. (2007). Brain correlates of self-rated originality of ideas: Evidence from event-related power and phase-locking changes in the EEG. Behavioral Neuroscience, 121, 224-230.

Hebb, D. O. (1949). The organization of behavior: A neuropsychological theory. New York: Wiley.

Hemmelmann, C., Horn, M., Süsse, T., Vollandt, R., \& Weiss, S. (2005). New concepts of multiple tests and their use for evaluating high-dimensional EEG data. Lournal of Neuroscience Methods, 142, 209-217.

Jasper, H. H. (1958). Report of the committee on methods of clinical examination in electroencephalography. Electroencephalography and Clinical Neurophysiology, 10, 371-375.

Jausovec, N., \& Jausovec, K. (2000). EEG activity during the performance of complex mental problems. International Journal of Psychopbysiology, 36, 73-88.

Jung-Beeman, M., Bowden, E. M., Haberman, J., Frymiare, J. L. Arambel-Liu, S., Greenblatt, R., et al. (2004). Neural activity when people solve verbal problems with insight. PLOS Biology, 2, E97.

Keil, A., Muller, M. M., Gruber, T., Wienbruch, C., Stolarova, M., \& Elbert, T. (2001). Effects of emotional arousal in the cerebral hemispheres: A study of oscillatory brain activity and event-related potentials. Clinical Neurophysiology, 112, 2057-2068.

Kihlstrom, J. F. (1995). The rediscovery of the unconscious. In H. Morowitz \& J. Singer (Eds.), The mind, the brain, and complex adaptive systems. Reading, MA: Addison-Wesley.

Kornhuber, H. H., \& Deecke, L. (1965). Changes in the brain potential in voluntary movements and passive movements in man: Readiness potential and reafferent potentials. Pflugers Archiv fur die Gesamte Physiologie des Menschen und der Tiere, 284, 1-17.

Kounios, J., Fleck, J. I., Green, D. L., Payne, L., Stevenson, J. L., Bowden, E. M., et al. (2008). The origins of insight in resting-state brain activity. Neuropsvchologia, 46, 281-291.

Krause, C. M., Pesonen, M., \& Hamalainen, H. (2007). Brain oscillatory responses during the different stages of an auditory memory search task in children. NeuroReport, 18, 213-216.

Libet, B., Gleason, C. A., Wright, E. W., \& Pearl, D. K. (1983). Time of conscious intention to act in relation to onset of cerebral activity (readiness-potential). The unconscious initiation of a freely voluntary act. Brain, 106, 623-642.

Luo, J., \& Niki, K. (2003). Function of hippocampus in "insight" of problem solving. Hippocampus, 13, 316-323.

Mai, X. Q., Luo, J., Wu, J. H., \& Luo, Y. J. (2004). “Aha!” effects in a guessing riddle task: An event-related potential study. Human Brain Mapping, 22, 261-270.

Mayer, R. E. (1992). Thinking, problem solving. New York: Freeman.
Metcalfe, J., \& Wiebe, D. (1987). Intuition in insight and noninsight problem solving. Memory \& Cognition, 15, 238-246.

Miller, L. A., \& Tippett, L. J. (1996). Effects of focal brain lesions on visual problem-solving. Neuropsychologia. 34, 387-398.

Morup, M., Hansen, L. K., \& Arnfred, S. M. (2007). ERPWAVELAB a toolbox for multi-channel analysis of time-frequency transformed event related potentials. Lournal of Neuroscience Methods, 161, 361-368.

Morup, M., Hansen, L. K., Herrmann, C. S., Parnas, J., \& Arnfred, S. M. (2006). Parallel factor analysis as an exploratory tool for wavelet transformed event-related EEG. Neuroimage, 29, 938-947.

Nicolaou, N., \& Nasuto, S. J. (2003). Comparison of temporal and standard independent component analysis (ICA) algorithms for EEG analysis. Paper presented at the ICANN/ICONIP'03, Joint 13th International Conference on Artificial Neural Networks and 10th International Conference on Neural Information Processing, Istanbul, Turkey.

Ohlsson, S. (1992). Information processing explanations of insight and related phenomena. In M. Keane \& K. Gillhooley (Eds.), Advances in the psychology of thinking (pp. 1-44). London: Harvester-Wheatsheaf.

Pesonen, M., Bjornberg, C. H., Hamalainen, H., \& Krause, C. M. (2006). Brain oscillatory 1-30 Hz EEG ERD/ERS responses during the different stages of an auditory memory search task. Neuroscience Letters, 399, 45-50.

Pesonen, M., Hamalainen, H., \& Krause, C. M. (2007). Brain oscillatory 4-30 Hz responses during a visual n-back memory task with varying memory load. Brain Research, 1138, 171-177.

Raghavachari, S., Kahana, M. J., Rizzuto, D. S., Caplan, J. B., Kirschen, M. P., Bourgeois, B., et al. (2001). Gating of human theta oscillations by a working memory task. Journal of Neuroscience, 21, 3175-3183.

Razumnikova, O. M. (2007). Creativity related cortex activity in the remote associates task. Brain Research Bulletin, 73, 96-102.

Schilling, M. A. (2005). A "small-world" network model of cognitive insight. Creativity Research Iournal. 17, 131-154.

Schooler, J. W., Fallshore, M., \& Fiore, S. M. (1995). Epilogue: Putting insight into perspective. In R. J. Sternberg \& J. E. Davidson (Eds.), The nature of insight. Cambridge, MA: MIT Press.

Seifert, C. M., Meyer, D. E., Davidson, N., Patalano, A. L., \& Yaniv, I. (1995). Demystification of cognitive insight: Opportunistic assimilation and the prepared-mind perspective. In R. J. Sternberg \& J. E. Davidson (Eds.), The nature of insight (pp. 65-124). Cambridge, MA: MIT Press.

Simonton, D. K. (1999). Creativity as blind variation and selective retention: Is the creative process Darwinian? Psychological Inquiry, 10, 309-328.

Sternberg, R. J., \& Davidson, J. E. (1995). The nature of insight. Cambridge, MA: The MIT Press.

Tallon-Baudry, C., \& Bertrand, O. (1999). Oscillatory gamma activity in humans and its role in object representation. Trends in Cognitive Sciences, 3, 151-162.

Terzis, G. (2001). How crosstalk creates vision-related eureka moments. Philosophical Psvchologv, 14, 393-421.

Weiss, S., \& Rappelsberger, P. (1998). Left frontal EEG coherence reflects modality independent language processes. Brain Topography, 11, 33-42.

Zeileis, A. (2004). Econometric computing with HC and HAC covariance matrix estimators. Journal of Statistical Software, 11, 1-17. 